\title{
Block pulse functions for solving fractional Poisson type equations with Dirichlet and Neumann boundary conditions
}

\author{
Jiaquan Xie ${ }^{1,3}$, Qingxue Huang ${ }^{1,2^{*}}$, Fuqiang Zhao ${ }^{1,3}$ and Hailian Gui ${ }^{1,3}$
}

\section{"Correspondence:}

tykjdxxjq@163.com

'College of Mechanical

Engineering, Taiyuan University of

Science and Technology, Taiyuan,

Shanxi 030024, China

${ }^{2}$ College of Mechanical Engineering,

Taiyuan University of Technology,

Taiyuan, Shanxi 030024, China

Full list of author information is

available at the end of the article

\begin{abstract}
In this study, the numerical technique based on two-dimensional block pulse functions (2D-BPFs) has been developed to approximate the solution of fractional Poisson type equations with Dirichlet and Neumann boundary conditions. These functions are orthonormal and have compact support on [0,1]. The proposed method reduces the original problems to a system of linear algebra equations that can be solved easily by any usual numerical method. The obtained numerical results have been compared with those obtained by the Legendre and CAS wavelet methods. In addition an error analysis of the method is discussed. Illustrative examples are included to demonstrate the validity and robustness of the technique.
\end{abstract}

Keywords: block pulse functions; fractional Poisson type equations; numerical solution; Dirichlet and Neumann boundary conditions; error analysis

\section{Introduction}

Fractional calculus is an important theoretical branch of mathematical theories [1], which has been widely applied in various fields such as the complex physical, mechanical, biological, and engineering fields. For example, fractional calculus has been applied to model the nonlinear oscillations of earthquake [2], fluid-dynamic traffic [3], continuum and statistical mechanics [4], signal processing [5], control theory [6] and dynamics of interfaces between nanoparticles and subtracts [7]. In these practical applications, fractional calculus has a certain geometric and physical meaning. Due to its historical dependence and globally related characteristics, fractional differentiation can be approximately represented and vigorously developed in the anomalous diffusion. In view of the fact that fractional calculus has great practical significance, it is very important to study fractional differential equations. In general, the exact solutions of many fractional partial differential equations cannot be obtained, so scholars are committed to obtaining their numerical solutions to reflect the exact solutions. In recent years, the theory of fractional calculus has been greatly developed, and lots of articles about fractional calculus have been published. Numerical algorithms for different types of fractional differential equations have been presented. These algorithms include the Chebyshev and Legendre polynomials method [8, 9], the wavelet method [10,11], the piecewise constant orthogonal functions method [12], the differential transform method [13], the collocation method [14], the Adomian decomposi-

(c) The Author(s) 2017. This article is distributed under the terms of the Creative Commons Attribution 4.0 International License (http://creativecommons.org/licenses/by/4.0/), which permits unrestricted use, distribution, and reproduction in any medium, provided you give appropriate credit to the original author(s) and the source, provide a link to the Creative Commons license, and indicate if changes were made. 
tion method [15], and so on. In [16], the authors proposed a compact exponential scheme for the time fractional convection-diffusion reaction equation with variable coefficients. In [11], the authors acquired the numerical solution of fractional Poisson equations using a two-dimensional Legendre wavelet. In [17], Maleknejad and Mahdiani proposed to solve nonlinear mixed Volterra-Fredholm integral equations with two-dimensional block pulse functions using a direct method. Reference [18] gave the numerical methods for solving two-dimensional nonlinear integral equations of fractional order by using a twodimensional block pulse operational matrix. In this study, we applied two-dimensional block pulse functions to obtain the numerical solutions of fractional Poisson type equations with Dirichlet and Neumann boundary conditions.

The current paper is organized as follows. In Section 2, the model with respect to Poisson type equations is given. In Section 3, some basic definitions and mathematical preliminaries of fractional calculus are introduced. Section 4 introduces the definitions and properties of two-dimensional block pulse functions. In Section 5, we discussed the error analysis of our approach. Section 6 introduces the method for solving fractional Poisson type equations. In Section 7, the proposed approach is tested through several numerical examples. Finally, a conclusion is given in Section 8.

\section{Illustration of the proposed model}

In the following, we introduce the propagation of an elastic wave for a one-dimensional rod model under the action of impact load.

The rod fixed at left and the right is exerted on a tensile impact load $P$, the length of it is $L$, as is shown in Figure 1. The basic equation of this problem with boundary condition is given as

$$
\left\{\begin{array}{l}
\frac{\partial^{2} u}{\partial x^{2}}-\frac{1}{c^{2}} \frac{\partial^{2} u}{\partial t^{2}}=0 \quad(0<x<L) \\
u(x, 0)=\dot{u}(x, L)=0, \quad u(0, t)=0, \quad \sigma(L, t)=P
\end{array}\right.
$$

where $c=\sqrt{E / p}$, is the wave velocity. The displacement analytical solution of the rod is

$$
\begin{aligned}
u(x, t)= & \frac{c}{E}\left\{\left[H\left(t+\frac{x-L}{c}\right)-H\left(t-\frac{x+L}{c}\right)\right]\right. \\
& -\left[H\left(t+\frac{x-3 L}{c}\right)-H\left(t-\frac{x+3 L}{c}\right)\right] \\
& \left.+\left[H\left(t+\frac{x-5 L}{c}\right)-H\left(t-\frac{x+5 L}{c}\right)\right]-\cdots\right\},
\end{aligned}
$$

where $H(x)$ is a Heaviside step function:

$$
H(x)= \begin{cases}1, & x>0 \\ 0, & x<0\end{cases}
$$

Figure 1 One-dimensional bar model under the impact load.

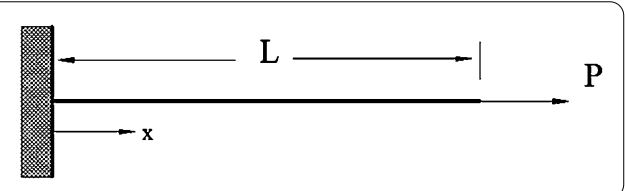


Based on the above model, we propose the following fractional Poisson equations model:

$$
\frac{\partial^{v} u}{\partial x^{\nu}}(x, t)+\frac{\partial^{\gamma} u}{\partial t^{\gamma}}(x, t)=g(x, t) \quad(0 \leq x \leq L, 0 \leq t \leq \tau)
$$

with Dirichlet boundary conditions

$$
\begin{cases}u(x, 0)=f_{0}(x), & u(0, t)=g_{0}(t) \\ u(x, \tau)=f_{1}(x), & u(L, t)=g_{1}(t)\end{cases}
$$

The Neumann boundary conditions are

$$
\begin{cases}u(x, 0)=f_{0}(x), & u(0, t)=g_{0}(t) \\ \left.\frac{\partial u}{\partial t}\right|_{(x, \tau)}=f_{1}(x), & \left.\frac{\partial u}{\partial x}\right|_{(L, t)}=g_{1}(t) .\end{cases}
$$

\section{Preliminaries and notations}

In this section, we gave some necessary definitions and preliminaries of the fractional calculus theory which will be used in the article [1].

Definition 1 The Riemann-Liouville fractional integral operator $I^{v}$ of order $v$ is given by

$$
\left(I^{v} f\right)(t)= \begin{cases}\frac{1}{\Gamma(v)} \int_{0}^{t}(t-\tau)^{v-1} f(\tau) \mathrm{d} \tau, & v>0 \\ f(t), & v=0\end{cases}
$$

Definition 2 The Caputo definition of fractional differential operator is given by

$$
{ }_{c} D^{v} f(t)= \begin{cases}\frac{1}{\Gamma(m-v)} \int_{0}^{t} \frac{f^{(m)}(\tau)}{(t-\tau)^{v-m+1}} \mathrm{~d} \tau, & 0<m-1 \leq v<m ; \\ \frac{\mathrm{d}^{m} f(t)}{\mathrm{d} t^{m}}, & v=m \in N^{+}\end{cases}
$$

For the Caputo derivative, we have

$$
{ }_{c} D^{v} t^{\gamma}= \begin{cases}0, & \text { for } \gamma \in N_{0} \text { and } \gamma<\lceil\nu\rceil ; \\ \frac{\Gamma(\gamma+1)}{\Gamma(\gamma+1-\nu)} t^{\gamma-\nu}, & \text { for } \gamma \in N_{0} \text { and } \gamma \geq\lceil\nu\rceil \text { or } \gamma \notin N_{0} \text { and } \gamma>\lfloor\nu\rfloor .\end{cases}
$$

\section{Two-dimensional block pulse functions}

One-dimensional block pulse functions have been widely used for differential and integral equations. More details for block pulse functions of the one-dimensional case are given in [19]. These conclusions can be extended to the two-dimensional block pulse functions.

\subsection{Definitions and properties}

2D-BPFs are defined as

$$
\phi_{i_{1}, i_{2}}(x, t)= \begin{cases}0, & \left(i_{1}-1\right) h_{1} \leq x<i_{1} h_{1} \text { and }\left(i_{2}-1\right) h_{2} \leq x<i_{2} h_{2}, \\ 1, & \text { otherwise }\end{cases}
$$

where $i_{1}=1,2, \ldots, m_{1}$ and $i_{2}=1,2, \ldots, m_{2}$ with positive integer values for $m_{1}, m_{2}$ and $h_{1}=\frac{T_{1}}{m_{1}}, h_{2}=\frac{T_{2}}{m_{2}}, T_{1}, T_{2} \in N^{+}$. 
They have the following properties:

1. Disjointness:

$$
\phi_{i_{1}, i_{2}}(x, t) \phi_{j_{1}, j_{2}}(x, t)= \begin{cases}\phi_{i_{1}, i_{2}}(x, t), & i_{1}=j_{1} \text { and } i_{2}=j_{2} \\ 0, & \text { otherwise. }\end{cases}
$$

2. Orthogonality:

$$
\int_{0}^{T_{1}} \int_{0}^{T_{2}} \phi_{i_{1}, i_{2}}(x, t) \phi_{j_{1}, j_{2}}(x, t) \mathrm{d} t \mathrm{~d} x= \begin{cases}h_{1} h_{2}, & i_{1}=i_{2} \text { and } j_{1}=j_{2} \\ 0, & \text { otherwise }\end{cases}
$$

In the region of $x \in\left[0, T_{1}\right)$ and $t \in\left[0, T_{2}\right)$, where $i_{1}, j_{1}=1,2, \ldots, m_{1}$ and $i_{2}, j_{2}=1,2, \ldots, m_{2}$.

3. Completeness: For every $u \in L^{2}\left(\left[0, T_{1}\right) \times\left[0, T_{2}\right)\right)$ when $m_{1}$ and $m_{2}$ approach infinity, Parseval's identity holds:

$$
\int_{0}^{T_{1}} \int_{0}^{T_{2}} u^{2}(x, t) \mathrm{d} t \mathrm{~d} x=\sum_{i_{1}=1}^{\infty} \sum_{i_{2}=1}^{\infty} u_{i_{1}, i_{2}}^{2}\left\|\phi_{i_{1}, i_{2}}(x, t)\right\|^{2}
$$

where

$$
u_{i_{1}, i_{2}}=\frac{1}{h_{1} h_{2}} \int_{0}^{T_{1}} \int_{0}^{T_{2}} u(x, t) \phi_{i_{1}, i_{2}}(x, t) \mathrm{d} t \mathrm{~d} x .
$$

\subsection{BPFs expansions}

A function $u(x, t) \in L^{2}\left(\left[0, T_{1}\right) \times\left[0, T_{2}\right)\right)$ can be expressed as

$$
u(x, t) \cong \sum_{i_{1}=1}^{m_{1}} \sum_{i_{2}=1}^{m_{2}} u_{i_{1}, i_{2}} \phi_{i_{1}, i_{2}}(x, t)=V^{T} \Phi(x, t),
$$

where

$$
\begin{aligned}
& V=\left[u_{11}, \ldots, u_{1, m_{2}}, \ldots, u_{m_{1}, 1}, \ldots, u_{m_{1}, m_{2}}\right]^{T} \\
& \Phi(x, t)=\left[\phi_{1,1}(x, t), \ldots, \phi_{1, m_{2}}(x, t), \ldots, \phi_{m_{1}, 1}(x, t), \ldots, \phi_{m_{1}, m_{2}}(x, t)\right]^{T} .
\end{aligned}
$$

The block pulse coefficients $u_{i_{1}, i_{2}}$ are obtained by

$$
u_{i_{1}, i_{2}}=\frac{1}{h_{1} h_{2}} \int_{\left(i_{1}-1\right) h_{1}}^{i_{1} h_{1}} \int_{\left(i_{2}-1\right) h_{2}}^{i_{2} h_{2}} u(x, t) \mathrm{d} t \mathrm{~d} x
$$

Since each of the two-dimensional block pulse functions takes only one value in its subregion, the $2 \mathrm{D}-\mathrm{BPF}$ can be expanded by the two $1 \mathrm{D}-\mathrm{BPF}$ :

$$
\phi_{i_{1}, i_{2}}(x, t)=\phi_{i_{1}}(x) \phi_{i_{2}}(t)
$$

where $\phi_{i_{1}}(x)$ and $\phi_{i_{2}}(t)$ are the $1 \mathrm{D}-\mathrm{BPF}$ related to the variables $x$ and $t$, respectively. Then we have

$$
u(x, t) \cong \Phi^{T}(x) U \Phi(t),
$$


where

$$
\begin{aligned}
& U=\left[\begin{array}{cccc}
u_{1,1} & u_{1,2} & \cdots & u_{1, m_{1}} \\
u_{2,1} & u_{2,2} & \cdots & u_{2, m_{1}} \\
\vdots & \vdots & \ddots & \vdots \\
u_{m_{1}, 1} & u_{m_{1}, 2} & \cdots & u_{m_{1}, m_{2}}
\end{array}\right], \\
& \Phi(x)=\left[\phi_{1}(x), \phi_{2}(x), \ldots, \phi_{m_{1}}(x)\right]^{T}, \quad \Phi(t)=\left[\phi_{1}(t), \phi_{2}(t), \ldots, \phi_{m_{2}}(t)\right]^{T} .
\end{aligned}
$$

\subsection{Operational matrix}

In this part, we may simply introduce the operational matrix of fractional integration of block pulse functions; more details can be found in [20].

Let $m_{1}=m_{2}=m$ and $T_{1}=T_{2}=T$. If $I^{\alpha}$ is the fractional integration operator of the block pulse functions, we can get

$$
I^{\alpha} \Phi(x) \cong F_{\alpha} \Phi(x)
$$

where

$$
F_{\alpha}=\left(\frac{T}{m}\right)^{\alpha} \frac{1}{\Gamma(\alpha+2)}\left[\begin{array}{ccccc}
1 & \xi_{1} & \xi_{2} & \cdots & \xi_{m-1} \\
0 & 1 & \xi_{1} & \cdots & \xi_{m-2} \\
0 & 0 & 1 & \cdots & \xi_{m-3} \\
\vdots & \vdots & \vdots & \ddots & \vdots \\
0 & 0 & 0 & \cdots & 1
\end{array}\right]
$$

and

$$
\xi_{k}=(k+1)^{\alpha+1}-2 k^{\alpha+1}+(k-1)^{\alpha+1} .
$$

$F_{\alpha}$ is called the block pulse operational matrix of fractional integration.

Let $D_{\alpha}$ is the block pulse operational matrix for the fractional differentiation. According to the property of fractional calculus, $D_{\alpha} F_{\alpha}=I$, we can easily obtain the matrix $D_{\alpha}$ by inverting the $F_{\alpha}$ matrix.

\section{Error analysis}

The error can be achieved when a function $u(x, t)$ is represented by 2D-BPFs over the region $D=[0, T) \times[0, T)$. Let $m_{1}=m_{2}=m$, so $h_{1}=h_{2}=\frac{T}{m}$.

We can define the error between $u(x, t)$ and its 2D-BPFs approximation, $u_{m}(x, t)$, over the sub-region $D_{i_{1}, i_{2}}$ as follows:

$$
e_{i_{1}, i_{2}}(x, t)=u_{i_{1}, i_{2}} \phi_{i_{1}, i_{2}}(x, t)-u(x, t)=u_{i_{1}, i_{2}}-u(x, t), \quad(x, t) \in D_{i_{1}, i_{2}},
$$

where

$$
D_{i_{1}, i_{2}}=\left\{(x, t) \mid \frac{i_{1}-1}{m} \leq x<\frac{i_{1}}{m}, \frac{i_{2}-1}{m} \leq t<\frac{i_{2}}{m}\right\} .
$$


Using the mean value theorem

$$
\left\|e_{i_{1}, i_{2}}\right\|^{2} \leq \frac{2}{m^{4}} M^{2}
$$

where $\left\|u^{\prime}(x, t)\right\| \leq M$. Then the error function $e(x, t)$ is defined as follows:

$$
e(x, t)=u_{m}(x, t)-u(x, t)
$$

By using equation (22), we have

$$
\|e(x, t)\|^{2} \leq \frac{2}{m^{2}} M^{2}
$$

hence $\|e(x, t)\|=O\left(\frac{1}{m}\right)$.

From equation (24), the conclusion is drawn that the method in this paper is convergent when it is used to solve the numerical solutions of fractional partial differential equations. For more details see [21].

\section{Description of the proposed method}

In the section, we will use the two-dimensional block pulse function to obtain the numerical solutions of equation (4) with Dirichlet and Neumann boundary conditions. We suppose $m_{1}=m_{2}=m$. Then we have

$$
\begin{aligned}
& \frac{\partial^{v} u}{\partial x^{v}} \cong \frac{\partial^{v}\left(\Phi^{T}(x) U \Phi(t)\right)}{\partial x^{v}}=\left[\frac{\partial^{v} \Phi(x)}{\partial x^{v}}\right] U \Phi(t)=\Phi^{T}(x) \mathbf{D}_{v}^{T} U \Phi(t), \\
& \frac{\partial^{\gamma} u}{\partial t^{\gamma}} \cong \frac{\partial^{\gamma}\left(\Phi^{T}(x) U \Phi(t)\right)}{\partial t^{\gamma}}=\Phi^{T}(x) U \frac{\partial^{\gamma}(\Phi(t))}{\partial t^{\gamma}}=\Phi^{T}(x) U \mathbf{D}_{\gamma} \Phi(t) .
\end{aligned}
$$

Using equation (18), the function $g(x, t)$ of equation (4) can be expressed as

$$
g(x, t) \cong \Phi^{T}(x) G \Phi(t)
$$

where $G=\left(g_{i j}\right)_{m \times m}$. Substituting equations (25)-(27) into equation (4), we have

$$
\Phi^{T}(x) \mathbf{D}_{v}^{T} U \Phi(t)+\Phi^{T}(x) U \mathbf{D}_{\gamma} \Phi(t)=\Phi^{T}(x) G \Phi(t)
$$

and dispersing equation (28) by the points $\left(x_{i}, t_{j}\right)(i, j=1,2, \ldots, m)$, we obtain

$$
\mathbf{D}_{v}^{T} U+U \mathbf{D}_{\gamma}=G
$$

which is a Sylvester equation.

(i) Dirichlet boundary conditions: For equation (4) with boundary conditions of equation (5), we have

$$
\begin{array}{ll}
\Phi^{T}(x) U \Phi(0) \approx \Phi^{T}(x) C_{1}, & \Phi^{T}(0) U \Phi(t) \approx C_{3}^{T} \Phi(t), \\
\Phi^{T}(x) U \Phi(\tau) \approx \Phi^{T}(x) C_{2}, & \Phi^{T}(L) U \Phi(t) \approx C_{4}^{T} \Phi(t) .
\end{array}
$$


The entries of vector $\Phi^{T}(x)$ and $\Phi(t)$ are independent, so from equation (30) we can obtain

$$
\begin{array}{ll}
\Lambda_{1}=U \Phi(0)-C_{1} \approx 0, & \Lambda_{3}=\Phi^{T}(0) U-C_{3}^{T} \approx 0, \\
\Lambda_{2}=U \Phi(\tau)-C_{2} \approx 0, & \Lambda_{4}=\Phi^{T}(L) U-C_{4}^{T} \approx 0,
\end{array}
$$

(ii) Neumann boundary conditions: Similar to equation (31), we can obtain

$$
\begin{aligned}
& \Lambda_{1}=U \Phi(0)-C_{1} \approx 0, \quad \Lambda_{3}=\Phi^{T}(0) U-C_{3}^{T} \approx 0, \\
& \Lambda_{2}=U \mathbf{D}_{1} \Phi(\tau)-C_{2} \approx 0, \quad \Lambda_{4}=\Phi^{T}(L) \mathbf{D}_{1}^{T} U-C_{4}^{T} \approx 0,
\end{aligned}
$$

equation (29) together with equation (31) or equation (32) gives a system of linear equations, we apply the least-square-method to solve the system, then the unknown function can be found.

\section{Numerical experiments}

In this section, we applied several numerical examples to test our proposed method, and compared the obtained numerical results with those obtained by the Legendre and the CAS wavelet methods.

Example 1 Consider the following Laplace equation:

$$
\frac{\partial^{v} u(x, t)}{\partial x^{v}}+\frac{\partial^{\gamma} u(x, t)}{\partial t^{\gamma}}=0
$$

with Dirichlet boundary conditions $u(x, 0)=0, u(0, t)=0, u(x, 1)=\sinh (x) \sin (1), u(1, t)=$ $\sinh (1) \sin (t)$. The exact solution of this problem for $v=\gamma=2$ is $u(x, t)=\sinh (x) \sin (t)$. The graph of the exact solution is shown in Figure 2. The graphs of numerical solutions for $m=8,16,32$ are shown in Figures 3, 4, 5 .

Example 2 Consider the following fractional Poisson equation:

$$
\frac{\partial^{4 / 3} u}{\partial x^{4 / 3}}+\frac{\partial^{3 / 2} u}{\partial t^{3 / 2}}=3 x^{2 / 3} t(t-2) / \Gamma\left(\frac{2}{3}\right)+4 \sqrt{t} x(x-2) / \sqrt{\pi},
$$

Figure 2 Exact solution for Example 1.

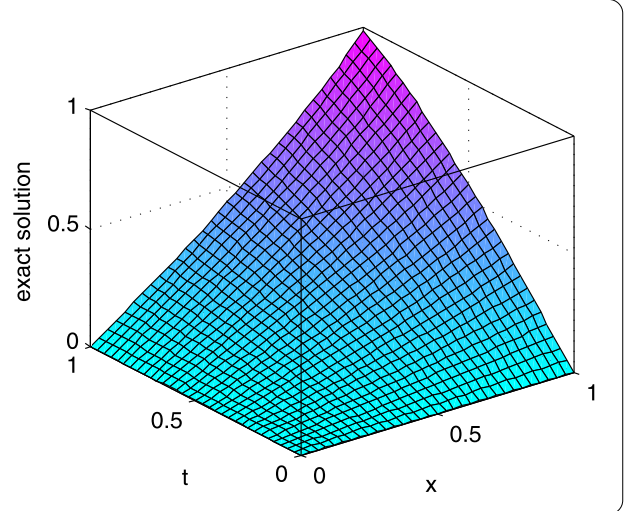


Figure 3 Numerical solution of $m=8$ for Example 1.

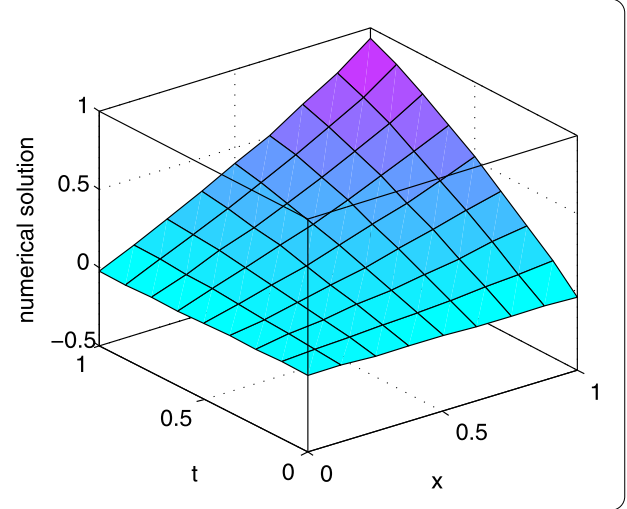

Figure 4 Numerical solution of $m=16$ for Example 1.



Figure 5 Numerical solution of $m=32$ for Example 1.

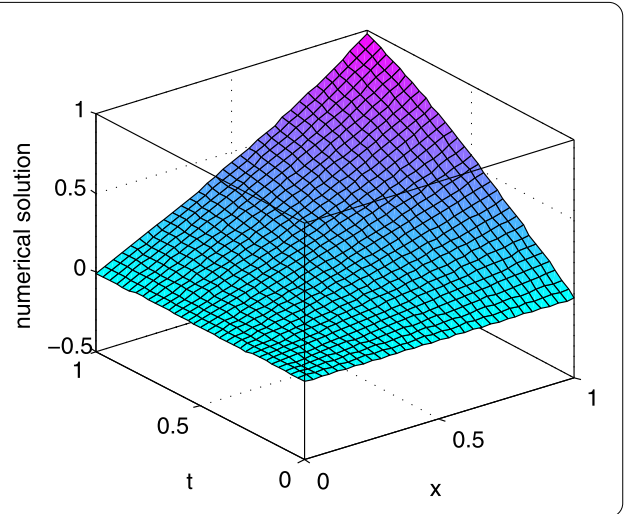

with homogeneous boundary conditions $u(x, 0)=u(x, 2)=u(0, t)=u(2, t)=0$. The analytical solution of this problem is $u(x, t)=x t(x-2)(t-2)$. The graph of exact solution is shown in Figure 6. The graphs of numerical solutions for $m=16,32,64$ in some nodes $(x, t) \in[0,2] \times[0,2]$ are shown in Figures 7, 8, 9. From Examples 1 and Examples 2, it can be concluded that the numerical solutions approximate the exact solutions well as $m$ grows.

Example 3 Consider the following Poisson equation:

$$
\frac{\partial^{v} u(x, t)}{\partial x^{v}}+\frac{\partial^{\gamma} u(x, t)}{\partial t^{\gamma}}=x\left(6+x^{2}\right) e^{-t},
$$


Figure 6 Exact solution for Example 2.

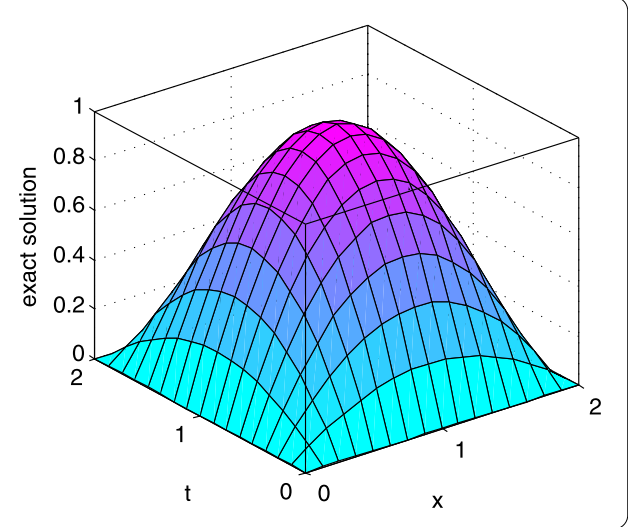

Figure 7 Numerical solution of $m=16$ for Example 2.

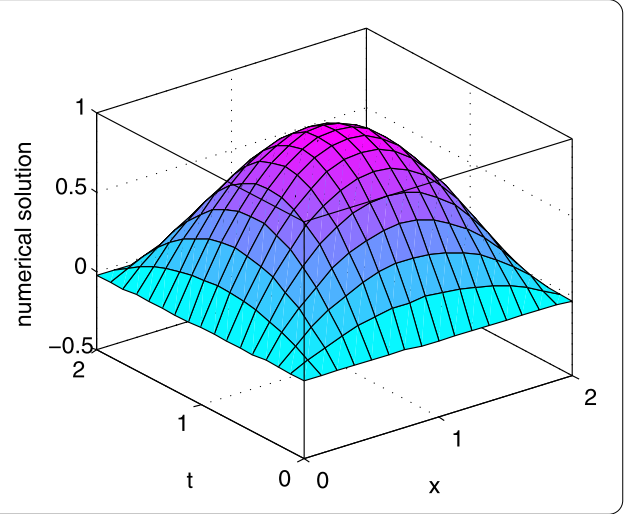

Figure 8 Numerical solution of $m=32$ for Example 2.

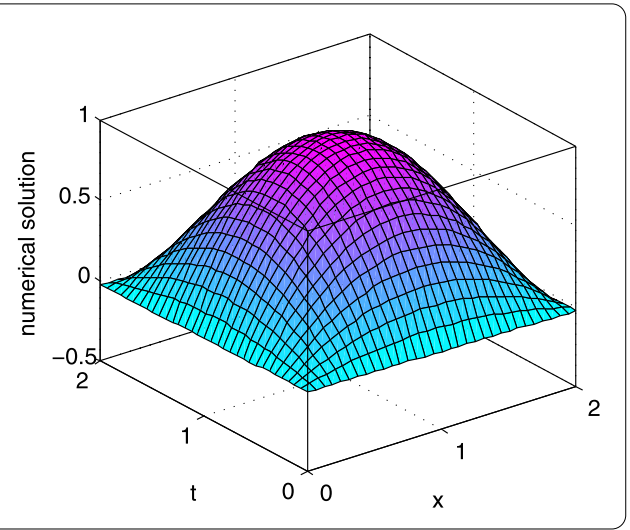

with the Neuman boundary condition $u(x, 0)=x^{3}, u(0, t)=0, \frac{\partial u(x, 1)}{\partial t}=-x^{3} e^{-1}, \frac{\partial u(1, t)}{\partial x}=3 e^{-t}$. The analytical solution of this problem for $v=\gamma=2$ is $u(x, t)=x^{3} e^{-t}$. Numerical results for some values of $\nu, \gamma$ and $m$ are listed in Table 1 . The contrastive graphs for our method, the Legendre and the CAS wavelet methods for $m=16,32,64$ are shown in Figures 10, 11, 12 . Table 1 shows that the numerical solutions are close to the analytical solution with $m$ increasing and the fractional order of $v, \gamma$ approaches 2 . From Figures 10, 11, 12, it can be seen that our approach has a great advantage over CAS wavelet method. Moreover, when $m=64$, our method can achieve the same satisfactory results as the Legendre wavelet method. 
Figure 9 Numerical solution of $m=64$ for Example 2.

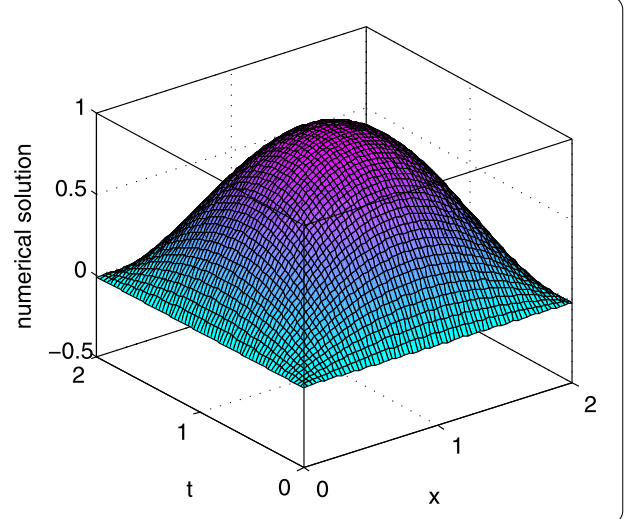

Table 1 Numerical results of some values of $v, \gamma$ and $m$ for Examples 3

\begin{tabular}{|c|c|c|c|c|c|}
\hline \multirow[t]{2}{*}{$(x, t)$} & \multicolumn{2}{|c|}{$v=2, \gamma=1.8$} & \multicolumn{2}{|c|}{$v=2, \gamma=1.9$} & \multirow[t]{2}{*}{ Exact solution } \\
\hline & $m=32$ & $m=64$ & $m=32$ & $m=64$ & \\
\hline$(0.2,0.2)$ & 0.0080999 & 0.0078027 & 0.0072661 & 0.0068916 & 0.0065498 \\
\hline$(0.4,0.4)$ & 0.0451791 & 0.0443244 & 0.0442091 & 0.0440639 & 0.0429004 \\
\hline$(0.6,0.6)$ & 0.1254325 & 0.1229019 & 0.1221991 & 0.1208139 & 0.1185433 \\
\hline$(0.8,0.8)$ & 0.2421242 & 0.2382542 & 0.2363440 & 0.2339913 & 0.2300564 \\
\hline$(1.0,1.0)$ & 0.4533293 & 0.3819264 & 0.3791327 & 0.3750676 & 0.3678794 \\
\hline
\end{tabular}
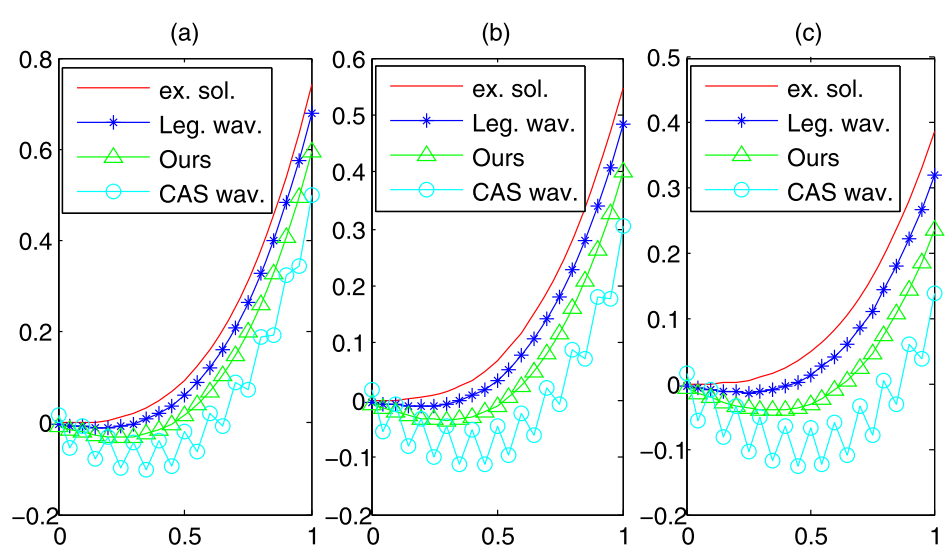

Figure 10 When $m=16$, the numerical solutions obtained by our method and those obtained by the Legendre and CAS wavelet methods at $t=0.3,0.6,0.95$; see (a), (b), and (c).

Example 4 Consider equation (35) with Neumann boundary conditions $u(x, 0)=0$, $u(0, t)=0, \frac{\partial u(x, 2)}{\partial t}=4 x^{2}, \frac{\partial u(2, t)}{\partial x}=4 t^{2}$. The analytical solution of this problem for $v=\gamma=\frac{3}{2}$ is $u(x, t)=x^{2} t^{2}$. Numerical results obtained by our method for $m=16,32,64,128$ in some nodes $(x, t) \in[0,2] \times[0,2]$ are listed in Table 2 . In the processor of Inter $(\mathrm{R})$ Core $(\mathrm{TM})$ i3-4150 CPU @ 3.50 Hz, RAM 4.00 GB and 32 bit Windows operating system, we use MATLAB R2010b to obtain the computing results and computing time for our method and the Legendre wavelet method. The contrastive graph of computing time is shown in Figure 13 (unit: second). Figure 13 shows that our method consumed less computing time than the Legendre wavelet method. This example aims to illustrate that our method has the advantages of easy theoretical construction and being less time consuming. 


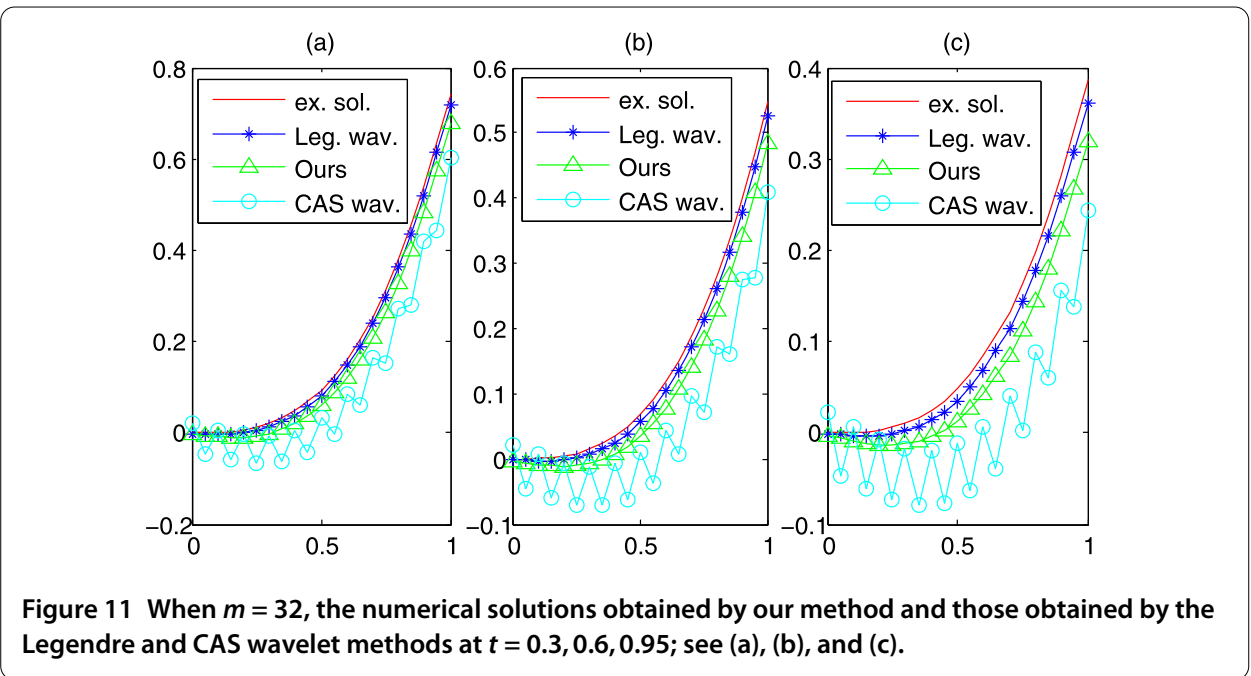

Legendre and CAS wavelet methods at $t=0.3,0.6,0.95$; see (a), (b), and (c).

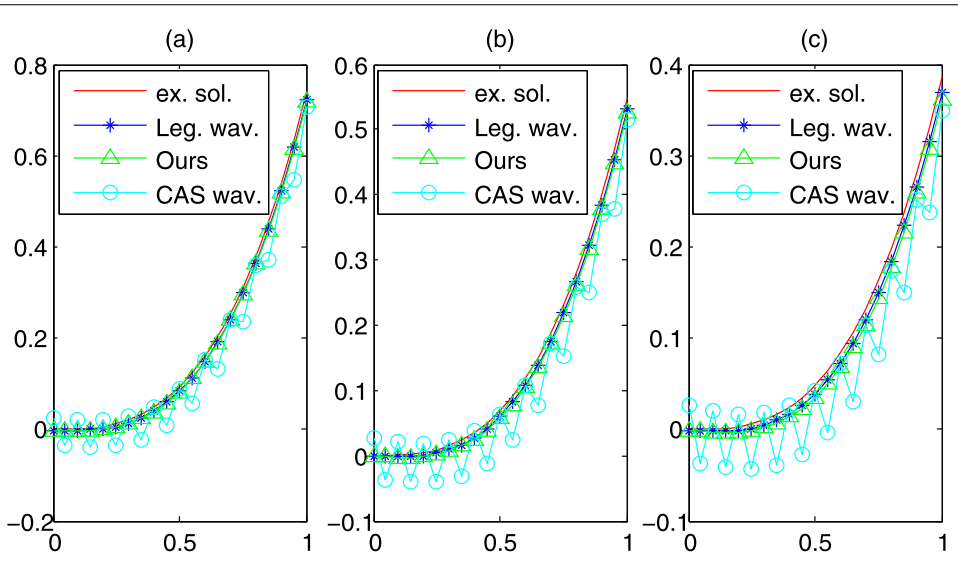

Figure 12 When $m=64$, the numerical solutions obtained by our method and those obtained by the Legendre and CAS wavelet methods at $t=0.3,0.6,0.95$; see (a), (b), and (c).

Table 2 Numerical results of different $\boldsymbol{m}$ for Examples 4

\begin{tabular}{lccccc}
\hline $\boldsymbol{( x , \boldsymbol { t } )}$ & Exact solution & $\boldsymbol{m}=\mathbf{1 6}$ & $\boldsymbol{m}=\mathbf{3 2}$ & $\boldsymbol{m}=\mathbf{6 4}$ & $\boldsymbol{m}=\mathbf{1 2 8}$ \\
\hline$(0,0)$ & 0 & 0 & 0 & 0 & 0 \\
$(1 / 4,1 / 4)$ & 0.0039 & 0.0036 & 0.0037 & 0.0039 & 0.0039 \\
$(2 / 4,2 / 4)$ & 0.0625 & 0.0620 & 0.0623 & 0.0625 & 0.0625 \\
$(3 / 4,3 / 4)$ & 0.3164 & 0.3159 & 0.3160 & 0.0362 & 0.3164 \\
$(1,1)$ & 1.0000 & 0.9996 & 0.9998 & 0.9999 & 1.0000 \\
$(5 / 4,5 / 4)$ & 2.4414 & 2.4406 & 2.4409 & 2.4413 & 2.4414 \\
$(6 / 4,6 / 4)$ & 5.0625 & 5.0618 & 5.0620 & 5.0624 & 5.0625 \\
$(7 / 4,7 / 4)$ & 9.3789 & 9.3776 & 9.3780 & 9.3785 & 9.3789 \\
$(2,2)$ & 16.0000 & 15.9987 & 15.9990 & 15.9996 & 15.9999 \\
\hline
\end{tabular}

\section{Conclusion}

In the present analysis, we have applied two-dimensional block pulse functions to approximate the solutions of Poisson type equations with two kinds of boundary conditions. Using this method, the system of fractional partial differential equations has been reduced to solving a system of algebraic equations. The accuracy of the solutions of the original problems will be improved using suitable $m_{1}$ and $m_{2}$. The block pulse functions are orthogonal 
Figure 13 The computing time consumed by our method and that consumed by the Legendre wavelet method.

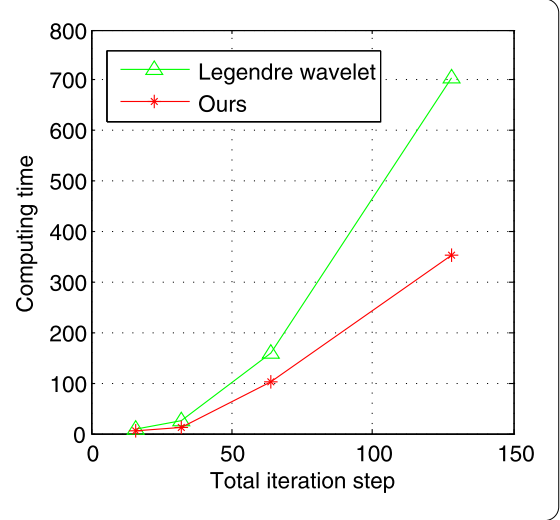

piecewise continuous functions which prompts flexibility for applications. Compared with other numerical methods, our proposed method has the great advantages of easy theoretical construction and being less time consuming. Additionally, the illustrated examples analyze and justify the ability and the reliability of the proposed method.

Competing interests

The authors declare that they have no competing interests.

Authors' contributions

JQX carried out the study. QXH, FQZ, and HLG supervised the work. JQX proposed the method for the analysis and all authors conducted the analysis. JQX prepared the manuscript under the guidance of QXH, FQZ, and HLG. FQZ and HLG provided the support of funds. All authors read and approved the final manuscript.

\section{Author details}

${ }^{1}$ College of Mechanical Engineering, Taiyuan University of Science and Technology, Taiyuan, Shanxi 030024, China. ${ }^{2}$ College of Mechanical Engineering, Taiyuan University of Technology, Taiyuan, Shanxi 030024, China. ${ }^{3}$ Shanxi Provincial Key Laboratory of Metallurgical Device Design Theory and Technology, Taiyuan, Shanxi, China.

\section{Acknowledgements}

This work was supported by the Natural Science Foundation of Shanxi Province (201601D011051), and the Dr. Startup funds of Taiyuan University of Science and Technology (20122054).

\section{Publisher's Note}

Springer Nature remains neutral with regard to jurisdictional claims in published maps and institutional affiliations.

Received: 2 December 2016 Accepted: 2 March 2017 Published online: 14 March 2017

\section{References}

1. Podlubny, I: Fractional Differential Equations. Academic press, San Diego (1999)

2. $\mathrm{He}, \mathrm{JH}$ : Nonlinear oscillation with fractional derivative and its applications. In: International Conference on Vibrating Engineering, pp. 288-291 (1998)

3. He, JH: Some applications of nonlinear fractional differential equations and their approximations. Bull. Sci. Technol. 15(2), 86-90 (1999)

4. Carpinteri, A, Mainardi, F: Fractals and Fractional Calculus in Continuum Mechanics, pp. 291-348. Springer, New York (1997)

5. Arqub, OA, El-Ajou, A, Momani, S: Constructing and predicting solitary pattern solutions for nonlinear time-fractional dispersive partial differential equations. J. Comput. Phys. 293, 385-399 (2015)

6. Bohannan, GW: Analog fractional order controller in temperature and motor control applications. J. Vib. Control 14(9-10), 1487-1498 (2008)

7. Vera, J, Bayazitoglu, Y: Temperature and heat flux dependence of thermal resistance of water/metal nanoparticle interfaces at sub-boiling temperatures. Int. J. Heat Mass Transf. 86, 433-442 (2015)

8. Yang, C: Chebyshev polynomial solution of nonlinear integral equations. J. Franklin Inst. 349(3), 947-956 (2012)

9. Chen, Y, Sun, Y, Liu, L: Numerical solution of fractional partial differential equations with variable coefficients using generalized fractional-order Legendre functions. Appl. Math. Comput. 244, 847-858 (2014)

10. Wang, L, Ma, Y, Meng, Z: Haar wavelet method for solving fractional partial differential equations numerically. Appl. Math. Comput. 227, 66-76 (2014)

11. Heydari, MH, Hooshmandasl, MR, Ghaini, FMM, et al.: Two-dimensional Legendre wavelets for solving fractional Poisson equation with Dirichlet boundary conditions. Eng. Anal. Bound. Elem. 37(11), 1331-1338 (2013) 
12. Yi, M, Huang, J, Wei, J: Block pulse operational matrix method for solving fractional partial differential equation. Appl. Math. Comput. 221, 121-131 (2013)

13. Momani, S, Odibat, Z, Erturk, VS: Generalized differential transform method for solving a space- and time-fractional diffusion-wave equation. Phys. Lett. A 370(5), 379-387 (2007)

14. Ma, X, Huang, C: Spectral collocation method for linear fractional integro-differential equations. Appl. Math. Model. 38(4), 1434-1448 (2014)

15. El-Kalla, IL: Convergence of the Adomian method applied to a class of nonlinear integral equations. Appl. Math. Lett. 21(4), 372-376 (2008)

16. Cui, M: Compact exponential scheme for the time fractional convection-diffusion reaction equation with variable coefficients. J. Comput. Phys. 280, 143-163 (2015)

17. Maleknejad, K, Mahdiani, K: Solving nonlinear mixed Volterra-Fredholm integral equations with two dimensional block-pulse functions using direct method. Commun. Nonlinear Sci. Numer. Simul. 16(9), 3512-3519 (2011)

18. Najafalizadeh, S, Ezzati, R: Numerical methods for solving two-dimensional nonlinear integral equations of fractional order by using two-dimensional block pulse operational matrix. Appl. Math. Comput. 280, 46-56 (2016)

19. Jiang, ZH, Schaufelberger, W: Block Pulse Functions and Their Applications in Control Systems. Springer, Berlin (1992)

20. Li, Y, Sun, N: Numerical solution of fractional differential equations using the generalized block pulse operational matrix. Comput. Math. Appl. 62(3), 1046-1054 (2011)

21. Maleknejad, K, Sohrabi, S, Baranji, B: Application of 2D-BPFs to nonlinear integral equations. Commun. Nonlinear Sci. Numer. Simul. 15(3), 527-535 (2010)

\section{Submit your manuscript to a SpringerOpen ${ }^{\mathcal{O}}$ journal and benefit from:}

- Convenient online submission

- Rigorous peer review

- Immediate publication on acceptance

- Open access: articles freely available online

- High visibility within the field

- Retaining the copyright to your article 